\title{
¿Hacia dónde va la ventaja competitiva?: Un modelo de gestión que incorpora a las personas como elemento clave de la estrategia empresarial
}

\author{
Emilio García Vega \\ Estudios de Maestría en Administración en la Universidad del Pacífico. \\ Profesor e Investigador del Departamento Académico de Administración de la \\ Universidad del Pacífico, Lima, Perú.
}

\begin{abstract}
Resumen
El concepto de ventaja competitiva ha evolucionado de manera acelerada en los últimos años. Algunos académicos y ejecutivos sostienen que las personas son el elemento fundamental para la construcción de la misma. En esta línea, la gestión de las empresas ha mostrado una inclinación hacia el manejo de los recursos humanos -denominados también «talentos» - como elemento clave de su éxito organizacional. En este trayecto, las ideas, los paradigmas y las concepciones se han modificado de manera interesante. Este texto trata de plantear estas nuevas concepciones frente al reto de gestionar una organización, y propone un modelo de gestión basado en la importancia de las personas en la administración de la ventaja competitiva.
\end{abstract}

\section{Palabras clave}

Estrategia, Ventaja Competitiva, Modelo de gestión, Gestión de personas

\section{Introducción}

Una gran preocupación en la gestión organizacional es el desarrollo de estrategias empresariales exitosas y, dentro de este ámbito, la construcción de ventajas competitivas saludables y verdaderamente estratégicas. Estos temas concitan una gran atención del quehacer gerencial actual.

La ventaja competitiva, que inicialmente partió de atributos fundamentalmente tangibles, con el trascurso de los años ha ingresado al terreno de la intangibilidad. Es en esta línea que las personas motivadas, acertadamente lideradas y que trabajan en equipo, empujan a las organizaciones al logro de los objetivos estratégicos por medio de estrategias empresariales implementadas de forma exitosa. Así, la manifestación de las personas como un «activo estratégico» importante resulta un hecho que ha cobrado gran relevancia en los últimos años. Es útil, en esta línea, citar a Jean-Jacques Servan-Schreiber ${ }^{1}$, quien afirmó que «la administración es a fin de cuentas, la más creadora de todas las artes. Es el arte de las artes; puesto que es el arte de organizar el talento».

1. Periodista, ensayista y político francés, fallecido recientemente (Fuente: http://www.elpais.com Fecha de consulta: 15/08/2008). 


\section{Objetivo}

El presente artículo tiene como objetivo plantear un modelo de gestión para el logro de la ventaja competitiva tomando como elemento fundamental a las personas.

\section{Metodología}

La metodología para la construcción del modelo de gestión que se plantea, se basa en el desarrollo de los siguientes elementos:

1. Ideas eje. Se trata de una serie de ideas que se consideran como elementales para asumir el reto de construir una ventaja competitiva y lograrlo, mediante el aporte de las personas en sus organizaciones. Estas ideas implican la ruptura o quiebre de ciertos «antiguos paradigmas»(Barker 1998) o «verdades fundamentales» que forman parte de la gerencia actual. No es posible hablar de una nueva visión en el desarrollo y mantenimiento de la ventaja competitiva, si no se parte de asumir nuevas «ideas eje» o lineamientos.

2. Puntos clave. Son planteamientos que se sugiere asumir frente al desarrollo de la ventaja competitiva en función de las personas.

3. Aspectos por gerenciar. En esta parte se consideran los elementos que tendrá en cuenta la persona que asuma el reto de lograr la ventaja competitiva de una organización y lo que demanda el manejo de las personas involucradas. Se trata de una gama de temas que estarán presentes en la labor gerencial.

4. ¿Cómo gerenciar eficientemente a las organizaciones del nuevo milenio? En este punto se emplean los elementos desarrollados en los tres puntos anteriores para tratar de responder a este interrogante. Se quiere establecer una opción que se considera viable, dada la actualidad empresarial.
Marco conceptual: Una forma de gestionar la ventaja competitiva tomando en cuenta a las personas

Un esquema y resumen del modelo planteado se puede observar en el anexo 1. A continuación, se procede a su presentación en detalle.

\section{Ideas eje}

Idea No 1: La importancia de la visión, la misión y los valores

La visión (el gran sueño de la organización), la misión (cómo se llegará a ese sueño) y los valores (elementos que respaldan y dan la pauta para el funcionamiento de las organizaciones), son piedras angulares sobre las cuales se construye la gestión de una organización. Es difícil avanzar sin saber a dónde se está yendo. Estos tres elementos dan las pautas para el establecimiento de los objetivos, la formulación de estrategias y la toma de decisiones organizacionales; sea en aspectos estratégicos, operativos o tácticos, es decir, desde los grandes temas hasta asuntos del día a día (David 2007).

Las respuestas a los comportamientos organizacionales, sean hacia el interior o hacia fuera de las organizaciones, se encuentran en estos tres aspectos. Por ello, se quiere recalcar la importancia de los conceptos mencionados en el párrafo anterior, que van a generar el «corazón», el «cerebro»y el «alma» de cualquier organización.

La historia puede brindar un testimonio relevante en lo que se refiere a la necesidad de la visión como piedra angular de una organización. Así, en la Edad Antigua, las legiones romanas, antes de emprender alguna batalla, decían a manera de «grito de batalla»: «Por Roma y por la gloria». De esta manera, esta civilización conquistó innumerables territorios y consolidó uno de los imperios más grandes de la historia de la humanidad².

2. Un análisis interesante acerca de las lecciones gerenciales que dejó la administración del Imperio Romano se puede revisar en Ortega, Antonio (2006). La antigua Roma: valores para el éxito empresarial. España: Pearson Educación S.A. 
Esta frase no es, propiamente en sí, una visión o una misión; sin embargo, encarna el propósito que empujaba a que los romanos emprendieran, lucharan y crecieran. Toda empresa debería buscar su propio «grito de batalla». La visión, la misión y los valores se constituyen en los medios para encontrarlo.

Idea № 2: La importancia de la selección de las personas

La parte administrativa de la gestión de personas resulta elemental para que cualquier iniciativa organizacional que se quiera aplicar resulte exitosa. La definición de un perfil adecuado a los retos que se quiera asumir es una labor prioritaria para la construcción de organizaciones modernas y con un «fuego interior» que las movilice. Las organizaciones deben preocuparse por contar con las personas adecuadas y no solo en lo que respecta a habilidades técnicas y a títulos universitarios, sino a la búsqueda de habilidades emocionales, que muchas veces son las que hacen la diferencia.

De lo anterior, puede derivar la siguiente pregunta: ¿qué se hace con el personal que ya estaba contratado en la empresa y que no ha sido seleccionado definiendo perfiles adecuados? En este sentido, se puede afirmar que la administración por competencias (Hellriegel y Jackson 2006) ofrece soluciones al respecto, ya que se pueden realizar estudios de los empleados para definir sus competencias, de tal manera que sean ubicados en el puesto que mejor se adecue a ellos. Es muy importante establecer que la ubicación se realice sobre la base de evaluaciones de este tipo; así, existe una mayor probabilidad de que el personal dé lo mejor de sí en su desempeño organizacional.

Idea N 3: El paso de la concepción de «recursos humanos» a «personas» $y$, luego, a «talentos» La concepción de los trabajadores ha ido evolucionando con el paso del tiempo. Ya no se puede considerar a la fuerza laboral únicamente como un recurso [recurso humano (Chiavenato 1990)], sino que se debe ir más allá. Primero, se puede afirmar que no se está tratando con recursos, sino que se está administrando gente, es decir, personas (Cantera y Gil 2006); y siendo más preciso aún, se está gestionando seres humanos. Esta concepción plantea que se debe tener en consideración todas las competencias relacionadas con la inteligencia emocional (Goleman y Chemiss 2001), que en muchas oportunidades son dejadas de lado por las gerencias. Además, las personas piensan, sienten, evolucionan y crecen como seres humanos. Una gestión eficiente debería considerar todos estos factores.

Sin embargo, esta evolución no queda allí. Se da un paso adicional y sumamente trascendental. Hace algunos años, existe la tendencia de afirmar que las personas son «talentos» (Chiavenato 2002). Esto quiere decir que la gestión no es de cualquier tipo de personas, sino que es de «personas talentosas». Se trata de elementos totalmente necesarios para el buen desempeño de la organización. Queda claro que este tipo de trabajadores no se encuentra en todos lados, razón por la cual las organizaciones deben crear el ambiente adecuado, cuando los encuentre, para que den lo mejor de sí en busca de la excelencia organizacional y personal (Peters y Austin 1987).

Idea $N^{\circ}$ 4: Las personas son fuente de ventaja competitiva

Michael Porter (1980) definió la estrategia competitiva como las acciones defensivas u ofensivas para crear una posición favorable en el mercado. Esta se basa en el desarrollo de una ventaja competitiva, entendiéndose por ella «el criterio básico de un desempeño más que regular a largo plazo» (Porter 2006: 11). Estos son dos conceptos básicos que todo estratega empresarial debe tomar en cuenta (Porter 1996). El concepto ha sido formulado, redefinido y discutido por académicos y pragmáticos, llegando a establecerse claramente su vigencia, la necesidad de estar reinventándola constantemente (Hamel y Prahalad 1996) y su adecuada gestión.

Linda Gratton (2001) explica el cambio de enfoque en lo que respecta a la concepción de la ven- 
taja competitiva. Explica cómo la antigua idea que establecía que los recursos financieros y tecnológicos eran las principales bases de las ventajas competitivas sostenibles, se ha transformado en un principio anacrónico. Gratton sostiene que las personas constituyen la fuente fundamental de este tipo de ventaja empresarial, tan buscada y venerada por los gerentes o gestores de proyectos que buscan ser eficientes y, por qué no decirlo, exitosos.

La evolución de las industrias ha llevado a ver cómo diversas ventajas han sido igualadas y rebasadas en diferentes ámbitos. Quizá a estas alturas del desarrollo humano y empresarial, se pueda afirmar que todo es posible de ser copiado. Acotando un poco más esta idea, se puede afirmar que contando con los capitales financieros adecuados, cualquier tecnología o know how podrían ser copiados. Entonces, la lógica podría llevar a que la ventaja va por este lado; sin embargo, en la actualidad existen diversas opciones de financiamiento a las que cualquier emprendedor, empresario o gerente con habilidad, criterio, manejo de información y conocimientos podría optar de manera ventajosa.

Es probable que en los países en vías de desarrollo, estas opciones de financiamiento aún no se plasmen en toda su magnitud. Sin embargo, al paso al que avanza nuestro sistema y sobre la base del proceso de globalización (Jeannet 2000) en el que se está inmerso, se puede decir que el tema de financiamiento para las empresas que demuestran cierta eficiencia en dicho manejo no es un tema limitante para su desarrollo (salvo evidencias explícitas de manejos financieros ineficientes o catástrofes empresariales).

Se cree, entones, que son las personas «aquello» que determinará la existencia de una ventaja competitiva $y$, acotando un poco más, se afirma que es la cultura organizacional, que se construye sobre la base de ellas, la que pondrá de manifiesto a esta ventaja (Breitfelder 2008).

Así, analizando estos aspectos como fuentes de ventaja competitiva se tiene que (Barney 2002): ¿́na. Las personas son únicas, cada uno de nosotros somos diferentes. Si una organización se gestiona de una manera adecuada, se logran grupos de talentos eficientes que, probablemente, resulten ganadores y exitosos. El grupo humano que conforma la organización es lo que la tornará en especial y única, debido a que la gestión adecuada de personas generará una cultura organizacional poderosa que hará diferente y mejor una institución dentro de una industria.

Đifícil de imitar. Lograr una cultura organizacional positiva y ganadora es muy difícil de imitar. Para ello, se debe haber trabajado tres aspectos básicos: el liderazgo, el trabajo en equipo y la motivación (intrínseca y extrínseca) (Nohria et al. 2008). Las organizaciones que logran manejar todos estos temas son inimitables. Esto se vuelve más estratégico aún, si se trata de una institución pionera (la primera en hacerlo) en una industria.

- Ectsosa de imitar. Los cambios en el manejo del personal y en el manejo de las culturas organizacionales tardan mucho tiempo, ya que implican modificaciones de modelos mentales. Cuando las empresas que compiten en una industria están rezagadas en estos temas, resulta muy costoso encarrilarse en esta línea. No se dice que no puedan lograrlo. Sin embargo, es muy probable que una empresa que ya está inmersa en la idea de que la ventaja competitiva son las personas, se encuentre en un estadio superior de desarrollo.

- Điferente. Las personas hacen diferentes a las organizaciones; es más, estas hacen a las organizaciones. Un grupo de personas, aunado a la visión, misión y valores a adecuados, logrará la combinación adecuada que la diferencie (una suerte de ADN organizacional) y que permitirá la construcción de una organización diferente y ganadora.

- Genera valor. Las personas positivamente gestionadas generan valor para todos los stakeholders (Freeman 1984) involucrados en el desarrollo de la organización. Generan valor para (Argandoña 1998): 
o Los accionistas (mayor retorno sobre su inversión).

o Ellos mismos (hacen cada vez mejores a sus organizaciones).

o El Estado (son contribuyentes ejemplares y ejemplo para la sociedad civil).

- Las comunidades con las que interactúan (son ciudadanos corporativos o empresariales ejemplares).

o El medio ambiente (asumen prácticas beneficiosas para el ecosistema que las acoge).

o Los proveedores [son transparentes con ellos («dan la cara») y establecen pautas de manejo adecuadas con ellos].

o La competencia (ponen a la ética empresarial como norma en todos sus actos).

Idea No 5: La competencia del futuro es cada vez más por intangibles, las personas son «extrañas» porque elevadas a la «n» pueden ser un intangible diferenciador $y$, a la vez, son las que gestionan esta nueva realidad.

La batalla por la eficiencia y el éxito empresarial ha rebasado los terrenos meramente tangibles y se ha proyectado a niveles intangibles y hasta etéreos. Hace algunos años, las empresas buscaban productos -la 'tangibilización' máxima era el producto y todas los elementos que emanaban de él- cada vez más innovadores y diferentes (Porter 2006). No se quiere decir que, en la actualidad, ya no exista una preocupación por estos. Sin embargo, lo que sucede es que las organizaciones ya no solo se preocupan por lo visible, sino que van más allá. En un inicio, la preocupación se centró en temas como la calidad (Bueno 1992: 71-78), el cual se fue cuantificando cada vez más con el paso del tiempo; luego se pasó a la gran preocupación por el posicionamiento (Ries 1992) (existe una «pelea» en la mente de los consumidores por posicionar conceptos y/o ideas).

Actualmente, las empresas han reforzado aun más esta última preocupación, ya que buscan ser buenos ciudadanos para que los consumidores, así como los potenciales consumidores, les tengan algún tipo de afecto y por qué no decirlo, hasta «cariño» (Roberts 2005). Es a este tipo de intangibilidad a la que se hace referencia. Es, entonces, entendible que se haya cambiado de paradigmas en lo que respecta a las bases de las ventajas competitivas.

Yendo más allá, la cultura (forma de hacer y pensar las cosas en las organizaciones) es un gran tema, que es aun más intangible. ¿No son acaso un buen producto, una eficiente campaña de marketing, un comercial efectivo y, en el fondo del asunto, una buena estrategia, claras manifestaciones de la cultura (Robbins 2004) de la empresa que las realiza?

Las personas son las que gestionan todo esto, de la sinergia del trabajo en equipo saldrán las estrategias que harán posible que las organizaciones sean «bien vistas», «queridas» $\mathrm{y}$ «deseadas» (que se posicionen positivamente en la mente de los consumidores-clientes). Para lograr la sinergia se necesita una gestión que busque la excelencia en dicha administración. Son las personas la base de la ventaja y, además, son las que la gestionan.

Idea № 6: Las personas deben ser lo más importante para las empresas

Las empresas son creadas por y para las personas. Estas son las que llevan a cabo las operaciones en todo nivel, y su enfoque fundamental está orientado a servir a los clientes y/o consumidores. En esta lógica, se puede afirmar que las personas deben ser lo más importante para las empresas. No es razonable cuestionar esta afirmación, aunque en la práctica algunos gerentes actúan de manera tal que las personas parecen ser la última prioridad en el manejo de sus organizaciones.

\section{Puntos clave}

Punto $N^{\circ} 1$ : El modelo necesita una retroalimentación constante y está evolucionando

Toda gestión necesita una serie de reportes o actividades de retroalimentación, ya que solo de esta manera se puede tener alguna idea acerca de los avances y los problemas que se presenten en la 
aplicación de nuevas tácticas, políticas y estrategias. Enrumbar hacia una nueva manera de gestionar la ventaja competitiva es muy complicado. Así, la retroalimentación es un elemento a tener siempre en cuenta en cualquier proceso administrativo, aun más en uno tan crucial como el que está relacionado con las personas en las organizaciones.

En suma, se sugiere dedicarle el tiempo necesario y prudencial para definir un sistema de retroalimentación adecuado, de manera que la gestión se afine cada vez más. Esto se puede realizar mediante herramientas cuantitativas o cualitativas. Así, se sugieren las siguientes ideas (Pearce y Robinson 2004):

- Heamientas cuantitativas. Se puede optar por diseñar encuestas y aplicarlas, ya sea de manera directa o por la Internet (en vista de que las organizaciones, por lo general, manejan este tipo de comunicación). Estas encuestas pueden servir para evaluar aspectos previos a las mejoras por implementar en la gestión o luego de la implementación, para realizar un adecuado seguimiento.

- Herramientascualitativas. Se pueden desarrollar focus groups y entrevistas a profundidad, para conocer mejor las opiniones del personal sobre los temas relacionados con la gestión de personas en la organización. Estas herramientas pueden servir en un primer momento exploratorio o pueden emplearse de forma concluyente para evaluar la marcha de la gestión.

- Herramientas cuali-cuantitativas. Se pueden diseñar herramientas que combinen las anteriores y que se alimenten además de técnicas como la observación, la experimentación, entre otras.

- Herramientasno pautadas. El gestor de personas debe ser capaz de buscar retroalimentaciones informales por medio de almuerzos, conversaciones en los pasillos, reuniones $u$ otros. Asimismo, con un verdadero compromiso, debe escuchar con «los oídos, los ojos y el corazón», procesar la información y retroalimentar su gestión.
Punto $N^{\circ} 2$ : La dicotomía de lo burocrático ante lo orgánico

Mintzberg (1984) plantea que existen dos grandes frentes en el perfil de las estructuras organizaciones, dos puntos antagónicos que grafican claramente comportamientos organizaciones diferentes y hasta confrontacionales. Cabe mencionar dichos enfoques para el diseño de las estructuras de las organizaciones (Mintzberg 1991):

-Burocrático-Mecánico. Se basa en una estructura vertical, con una cultura rígida y plantea trabajos rutinarios. Configura organizaciones muy normadas y, por lo tanto, extremadamente formales. Por lo general, auspicia la competencia como forma de trabajo entre áreas y personas. Casi siempre es autocrática.

- Adhocrático-Orgánico. Se fundamenta en una estructura horizontal, con una cultura que se adapta a las situaciones. Plantea un trato, directo e informal, que se basa en la colaboración entre el personal. En la medida de lo posible, trata de ser democrática.

Los que dirigen las organizaciones tienen estos dos modelos extremos como insumos para la construcción de las estructuras de los entes que administran, con todas las connotaciones que de la aplicación de una o de la otra se derivan. La cultura organizacional estará signada totalmente con la elección de un modelo o del otro. Sin embargo, como en casi todo en la administración, puede ser que no se esté ante una situación que permita hacer una distinción clara y total. Es probable que, en la práctica, aparezcan mezclas de ambos modelos en la realidad empresarial. En estos casos, es el gerente el total responsable, ya sea por brindar las pautas para que la organización se configure de dicha manera o de llevar a cabo los cambios pertinentes que derivarán en el desarrollo de una organización a la medida de los retos previstos.

El modelo Adhocrático permite una transformación organizacional porque su definición encaja perfectamente con la concepción de las personas como ventaja competitiva. Así, se puede afir- 
mar que las organizaciones deberían apuntar hacia su realización o, mejor dicho, hacia la «adhocratización» de su estructura organizacional, en la medida que su propia coyuntura lo demande y permita.

Punto No 3: El compromiso del ápice estratégico Si no existe un compromiso firme y seguro del ápice estratégico (Mintzberg 1984) de la organización con las ideas planteadas -que ponen a la persona sobre toda las cosas en las organizaciones-, es muy probable que la gestión de personas no se perfile hacia la excelencia, la eficiencia y, por qué no decirlo, hacia la sobrevivencia de las organizaciones.

Los cambios son más fáciles de gestionar cuando la gerencia está convencida de la necesidad de llevarlos a cabo. Mientras esta «no la vea», resulta muy complicado avanzar hacia una gestión de personas de vanguardia.

Es decir, se ve la necesidad de que los gerentes actuales y del futuro asuman como principios de su gestión las «ideas-eje» planteadas en este texto. No se quiere exagerar en la importancia de los planteamientos de este texto, pero se cree fervorosamente en que, en el siglo que ese está iniciando, la gerencia debería tomar muy en cuenta a las mismas.

En el mundo existen empresas de vanguardia, que han construido su liderazgo -y en algunos casos, su sobrevivencia- empresarial en las industrias que compiten, sobre la base de la concepción que las personas son lo primero. Estas empresas exitosas se basaron en ideas compartidas que fluyeron por toda la organización de manera adecuada, pero que siempre partieron del ápice estratégico y de allí llegaron hasta la jerarquía menor. Como ejemplo de esto se puede mencionar a las siguientes empresas: SAS Institute, Soutwest Airlines, Bloomberg, Unilever, Procter and Gamble, Nike, Diesel, Wendy's, Herman Miller, Ikea, Hewlett Packard, entre otras. Casos más cercanos a nuestra realidad son: Ambev en Brasil, Falabella en Chile o Telmex en México. En el Perú podemos ver señales, en mayor o menor medida, de la aplicación de este nuevo paradigma en empresas como: Kimberly Clark, Wong, Interbank; Corporación Furukawa, Scotiabank, entre otras. En todos los casos mencionados, se recalca que la asunción de esta nueva forma de percibir a las personas en las realidades empresariales que sirven como semillas para cambiar o diseñar nuevos modelos de gestión, partió del ápice estratégico.

\section{Aspectos por gerenciar}

\section{Se está gerenciando personas}

Es elemental tener siempre en cuenta que se está gerenciando personas. Algunos de los hechos que se derivan de esto y que son más que trascendentales para gerenciar personas, se muestran a continuación:

Todas las personas somos diferentes. Es imposible encontrar dos personas iguales en cualquier ámbito que se quiera comparar. Así las estrategias, políticas, tácticas, entre otros, que resultaron con un grupo de personas, no necesariamente tendrán los mismos resultados con todos los grupos que se gerencian. Esto se hace más notorio cuando se trata de grupos de nacionalidades, ubicaciones geográficas y/o coyunturas diferentes.

tapersonas somos complejas. Parafraseando a Mintzberg (1991), se puede decir que las personas somos «extrañas bestias», cada una de ellas con sus propios problemas, competencias, conflictos, entre otras características.

tapersonas somos una mezcla de seres racionales, económicos y emocionales. Las personas pensamos, generamos retornos económicos, nos preocupamos por la parte material y, al mismo tiempo, somos un cúmulo de sentimientos y valores. La gerencia de personas está en problemas cuando estos temas entran en conflicto.

- tapersonas pueden ser impredecibles. La complejidad y la mezcla hacen que las personas puedan reaccionar de maneras impensables ante situaciones similares. La situación personal puede empujar a reacciones desbocadas, diferentes o, en su defecto, extremadamente acertadas. 
—apersonas tienen egos diferentes. La egolatría es un problema que puede estar presente en las organizaciones en mayor porcentaje de lo esperado. Se puede observar no solo en los niveles gerenciales, sino también en todas las jerarquías organizacionales. La presencia de egos puede tornar muy complicado el logro del trabajo en equipo, de la eficiencia del liderazgo $y$, por ende, de la motivación de las personas. - No todas las personas pueden trabajar en equipo. Es necesario conocer las competencias de las personas en las organizaciones. Se da el caso de individuos a quienes, por sus modelos mentales, les cuesta mucho trabajar con otros. En ese caso, lo mejor es identificarlos y ubicarlos, dentro de la estructura organizacional, en aquellas posiciones en donde pueden ser más productivos o involucrarlos en formas de trabajo grupales. En el extremo, se puede optar por su reubicación por medio del diálogo, de manera que se eviten conflictos y frustraciones personales y organizacionales.

- tapersonas tienen sus propios modelos mentales. Una de las labores más complicadas de la gestión de personas es construir un modelo organizacional sobre la base de modelos mentales personales. Englobarlos y engarzarlos, adecuadamente, es todo un reto y arte.

- tapersonas tienen metas diferentes. Las personas tienen prioridades y objetivos no necesariamente similares, que pueden llevar a que la organización no alcance la buscada sinergia.

Por lo tanto, la gestión de personas es un reto muy complejo (Hernández et al. 2006).

\section{Cultura, liderazgo, motivación y trabajo en equipo}

Se han definido los siguientes aspectos clave:

- Eultura. Forma de pensar y hacer las cosas dentro de la organización (Robbins 2004). La cultura la perfila el ápice estratégico, pero solo se puede construir con la colaboración de todas las personas. En este proceso es claro que se pueden modificar las ideas iniciales, razón por la cual las decisiones de las altas jerarquías organizacionales o de los fundadores de la institución tendrán, necesariamente, que variar. Crear una cultura demanda mucho tiempo, pero es LA BASE DE LA VENTAJA COMPETITIVA TOMANDO COMO REFERENCIA PRINCIPAL A LA GESTIÓN DE PERSONAS.

La cultura actúa como una suerte de «pegamento», que permitirá que la organización se mantenga unida y que funcione. Las organizaciones con culturas fuertes y positivas son las que se perfilarán como exitosas, eficientes y, por ende, sobrevivirán en el tiempo.

tiderzgo (Robbins 2004). Consiste en llevar a las personas a resultados que no lograrían de manera individual. El liderazgo no se debe relacionar con el «mesianismo», sino que debe relacionarse con la capacidad de lograr que las personas colaboren entre sí para lograr resultados, los cuales pueden ser tangibles o intangibles. Sin un liderazgo positivo, las organizaciones tenderían a desaparecer por inanición.

Si bien las bases del liderazgo pueden ser formales o informales, lo importante es que la gerencia las identifique y las aproveche para el bien de las organizaciones y de las personas que las conforman.

Motivación (Robbins 2004). Se trata del interés que las personas sienten hacia su trabajo y hacia el logro de los objetivos trazados. La motivación está directamente relacionada con la satisfacción en el trabajo.

Fundamentalmente, la motivación puede manejarse de manera extrínseca (material) o intrínseca (reconocimiento o intangibles). Se cree que se debe balancear ambos tipos para llegar a las técnicas de motivación más adecuadas, de acuerdo con la organización que se esté gestionando. El uso de la motivación dentro del modelo de gestión de una empresa puede tener resultados sumamente provechosos para aquella (Nohria et al. 2008).

- Frałjo en equipo (Blake 1989). Consiste en lograr que las personas actúen como unidad, que se aproveche la diversidad de opiniones, procedencias y formaciones para obtener mejores resultados y mejores tomas de decisiones. A pesar de que existen diferentes grupos de trabajo, se cree que la sensación de comuni- 
dad y pertenencia son las principales condiciones para que se presente, decidida y eficientemente, el trabajo en equipo.

Aspectos que alteran la eficiencia de la gestión de personas: el poder y la política

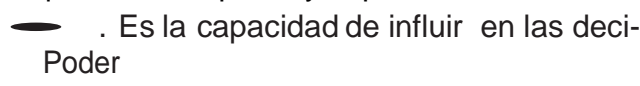

siones de la organización, de forma más puntual y humana, o la capacidad de conseguir lo que uno quiere (Robbins 2004).

Si el poder se emplea privilegiando intereses personales frente a los organizacionales, se puede tornar en un aspecto que impedirá el desarrollo organizacional, la eficiencia de un modelo de gestión basado en las personas y la puesta en marcha de cualquier iniciativa institucional.

El poder se debe emplear de manera positiva, buscando primordialmente el convencimiento antes que el sometimiento. Solo de esa manera se puede hablar de la construcción de una organización verdadera y no de una suma de individuos, realidades y poderes que se someten a un orden porque no tienen otra opción laboral o que, en el extremo, se retiran de la misma porque no tienen ninguna posibilidad de ser escuchados y/o desarrollarse.

- Política. Se puede definir a la política como una serie de elementos que surgen ante el descontento con la gestión organizacional. Se basa en la formación de grupos que ven la necesidad de ir contra lo establecido.

El caldo de cultivo para el surgimiento de la política en las organizaciones es la insatisfacción. La política es muy peligrosa porque puede destruir lo establecido y, aun más, puede ser más dañina cuando sirve a intereses personales. La política es una manera de llegar al poder.

Si existiera un proyecto organizacional compartido, la política puede ser vista como una enfermedad que corroe todo.

Aspectos que se busca lograr: aprendizaje y cambio A $m$ mizaje. Se puede afirmar que consiste en aprender a manejar situaciones. Lo ideal es que las organizaciones se construyan sobre la base de un aprendizaje limpio, alegre y pensado. En este sentido, alguien debería dar las pautas para lograr el aprendizaje organiza-cional, de manera que se establezcan las prioridades en función de la visión, la misión y los valores.

Eabio. Se puede aseverar que el cambio es el logro de modificaciones pensadas en las organizaciones, ante los retos que el entorno plantea. Todo puede cambiar en una organización, siempre y cuando se cuente con las personas adecuadas. Cabe mencionarque se puede optar por cambios en la cultura organizacional; sin embargo, estos no son inmediatos sino de largo plazo.

\section{¿Cómo se puede gerenciar eficientemente a las organizaciones del nuevo milenio?}

Claro está que nos encontramos ante un reto complicado, pero al mismo tiempo crucial, para que las empresas sobrevivan y sean exitosas en función de dar una mirada al pasado, presente y futuro. Se ha sostenido que las personas son una fuente de ventaja competitiva actual y futura, lo

que hace aun más trascendental la respuesta de esta interrogante. En este sentido, se ensayan los siguientes planteamientos ${ }^{3}$ :

\section{Forma No 1: Pensar antes de actuar}

En muchas oportunidades, se toman decisiones más por la información histórica que por la aplicación de un pensamiento organizacional y estratégico. En muchas empresas se actúa más por reflejo que por una cavilación real. Es claro que no se va a pensar ampliamente antes de realizar todos los procesos organizacionales; sin embargo, la labor gerencial debe estar signada por un PENSAMIENTO CUESTIONADOR frente a reacciones predefinidas.

Aquí, es donde el criterio del gestor desempeña un papel preponderante para responder pregun-

3. Aportes fundamentales en la visualización de las personas como ventaja competitiva de las organizaciones y, por ende, pilares en estos planteamientos del autor, son las ideas de Gratton $(2008,2001)$. 
tas cómo: ¿qué asuntos ameritan repensarlos?, ¿qué cuestionamientos son estratégicos? y ¿por qué ahora sí pensar y no actuar?

Cabe preguntarse: ¿cuánto tiempo se dedica a pensar en la organización? A pensar, por ejemplo, en nuevas estrategias, en nuevas formas de hacer las cosas, en nuevas ideas para mejorar el clima organizacional, en nuevas formas de lograr y celebrar los éxitos, entre otros.

Se cree que la principal tarea para gerenciar organizaciones es PENSAR. Solo tomando esa prerrogativa se puede estar un paso adelante en la industria y estar listo para dar el «gran salto» en la gestión de personas dentro de la organización y, aun más, en su gestión estratégica.

\section{Forma $N^{\circ}$ 2: Romper paradigmas}

La gestión está llena de paradigmas o afirmaciones que muchos gerentes consideran como dogmáticas. Una de ellas se ha tratado en este ensayo, la que afirma que las ventajas competitivas se generan sobre la base de recursos financieros y tecnológicos y no de las personas. Otra idea arraigada en la gerencia actual es el privilegio del corto plazo, y de los resultados frente al futuro y a los retos que este plantea.

Estos y otros paradigmas condicionan la actuación y, lo que es peor, el pensamiento de los gerentes actuales. La principal acción frente a esta realidad es que los gestores de las organizaciones deben tener la suficiente valentía de asumir la necesidad de cambiar, mutar y transgredir sus modelos mentales y optar por lo diferente, vanguardista y arriesgado, en la gestión. El objetivo es buscar el equilibrio perfecto entre las lecciones del pasado, la premura del presente y los retos del futuro.

\section{Forma No 3: Generar cultura}

Una empresa sin una cultura positiva y fuerte no puede ser eficiente ni podrá sobrevivir en el tiempo. La cultura es la base de todo, ya se ha dicho en este ensayo que cualquier manifestación de éxito empresarial es un reflejo de la cultura.
Construir una cultura organizacional implica un compromiso que supera lo inmediato y que apunta a ser lo suficientemente humilde para ser capaz de recibir retroalimentaciones de todo nivel jerárquico.

Lograr esa «forma de hacer y pensar las cosas en la organización» debe ser la principal preocupación de un gerente, ya sea en momentos de bonanza o de crisis. Una cultura organi-zacional positiva y sólida puede ser la diferencia entre sobrevivir y desaparecer, entre el éxito o la mediocridad organizacional, y entre ser «el lugar» para trabajar o ser simplemente un centro de trabajo en el que las personas ven que el tiempo pasa.

La cultura organizacional positiva puede permitir que el liderazgo, el trabajo y la motivación funcionen de manera adecuada y con un patrón claro, que evite conflictos y contradicciones.

\section{Forma $N^{\circ}$ 4: Aprender y Cambiar}

En muchas ocasiones, en las organizaciones se dan las cosas por oficializadas, normadas y sentadas. No existe una sensibilización a preocuparse por aprender y, sobre la base de esto, cambiar.

La trasgresión y lo contestatario parece no tener espacio en algunas organizaciones actuales. ¿Por qué cambiar las cosas, si todo parece funcionar bien? Se puede afirmar que los entornos siempre presentarán retos y que las empresas deben estar preparadas para asumirlos. Esto lo pueden lograr, si aprenden constantemente lo que les permitirá estar listas frente a los grandes cambios y, además, a manejarlos y por qué no a generarlos.

Aprender y cambiar son dos actividades que deben estar en la agenda de cualquier gerente que pretenda ser medianamente eficiente.

Forma N 5: Gestión del tiempo

El «tiempo organizacional» no es igual al «tiempo de las personas». Las empresas se mueven lentamente, razón por la cual los grandes cambios culturales nunca se desarrollarán en períodos cortos de tiempo. Se debe apuntar a equilibrar los resultados económicos inmediatos con la visión de lar- 
go plazo de las organizaciones. Suele ocurrir que los gerentes privilegien el logro personal (que se vea «su sello» en su gestión, por más corta que esta sea), en detrimento del proyecto organizacional al que se debe apuntar. Los egos también traicionan a los gerentes; incluso, pueden llegar a decidir «tirar el tablero», estableciendo nuevas estructuras de poder y alimentando la presencia de la política en las organizaciones.

Forma N 6: Generar el diálogo

Se está de acuerdo con Linda Gratton (2001), cuando afirma que «para crear un enfoque estratégico del personal es necesario un diálogo permanente en toda la organización». Este diálogo es estratégico para que las organizaciones puedan crecer, generar estrategias y generar cultura.

Las organizaciones deben preocuparse por establecer mecanismos, formales e informales, que permitan el logro del diálogo en todo nivel.
Análisis

Se puede afirmar que una muestra de la gestión exitosa de las personas, como fuente de ventaja competitiva, son las organizaciones que participan en las actividades y en la premiación denominada «Great Pleace to Work». Esta iniciativa cobra importancia día a día en el mundo y nuestro país no es ajeno a ello (en el anexo 2 se puede observar el listado de las 25 empresas que encabezan dicho ranking en el Perú).

A continuación se analiza brevemente (y en bloque) a las empresas del mencionado ranking sobre la base de información pública de las mismas. Se revisan las partes del modelo que se cree son factibles de evaluación; éstas son las dos primeras partes del mismo («ideas eje»y «puntos clave»). Frente a ellas, se formulan opiniones con respecto a los aspectos que son viables de apreciación.

Evaluación general del modelo planteado en las empresas del ranking del great place to work sobre la base de información pública de las mismas

\begin{tabular}{|c|c|c|}
\hline $\begin{array}{l}\text { Elementos del } \\
\text { modelo factibles } \\
\text { de evaluación }\end{array}$ & $\begin{array}{l}\text { Aspectos factibles de evaluación } \\
\text { sobre la base de información } \\
\text { pública de la empresa }\end{array}$ & EVALUACIÓN \\
\hline \multirow{3}{*}{ IDEAS EJE } & $\begin{array}{l}\text { La importancia de la visión, la mi- } \\
\text { sión y los valores }\end{array}$ & $\begin{array}{l}\text { Muy importante. En estas empresas todo gira en torno a la } \\
\text { Visión, Misión y los valores organizacionales. }\end{array}$ \\
\hline & $\begin{array}{l}\text { La importancia de la selección de } \\
\text { las personas }\end{array}$ & $\begin{array}{l}\text { Muy importante. Se trata de organizaciones con procesos de } \\
\text { selección bien planteados y llevados a cabo de forma adecuada. }\end{array}$ \\
\hline & $\begin{array}{l}\text { Las personas son fuente de venta- } \\
\text { ja competitiva }\end{array}$ & $\begin{array}{l}\text { Las personas son el eje - o un factor relevante - de la ventaja } \\
\text { competitiva de estas empresas. }\end{array}$ \\
\hline \multirow{4}{*}{ PUNTOS CLAVE } & $\begin{array}{l}\text { El modelo necesita una retroali- } \\
\text { mentación constante y está evolu- } \\
\text { cionando }\end{array}$ & $\begin{array}{l}\text { En la mayoría de estas organizaciones existen reuniones cons- } \\
\text { tantes de retroalimentación a diferentes niveles. }\end{array}$ \\
\hline & $\begin{array}{l}\text { La dicotomía de lo burocrático ante } \\
\text { lo orgánico }\end{array}$ & $\begin{array}{l}\text { A pesar de los diferentes rubros de estas organizaciones, existe } \\
\text { una tendencia hacia la horizontalidad y lo orgánico. }\end{array}$ \\
\hline & $\begin{array}{l}\text { El compromiso del ápice estraté- } \\
\text { gico }\end{array}$ & $\begin{array}{l}\text { Se puede apreciar que las gerencias generales están amplia- } \\
\text { mente involucradas con la concepción de las personas como } \\
\text { activos estratégicos de la empresa. }\end{array}$ \\
\hline & $\begin{array}{l}\text { Aspectos que se busca lograr: } \\
\text { aprendizaje y cambio }\end{array}$ & $\begin{array}{l}\text { En estas compañías se busca que sus trabajadores aprendan } \\
\text { constantemente y mejoren en su desempeño laboral. El cam- } \\
\text { bio es una variable siempre presente en la gestión de estas } \\
\text { empresas. }\end{array}$ \\
\hline
\end{tabular}

Fuente: Elaboración propia. 


\section{Conclusiones}

\section{La cultura organizacional es fundamental}

Se cree que la trilogía fundamental de la gestión de las organizaciones está dada por: la estrategia empresarial, la estructura organizacional y la cultura organizacional. Todo esfuerzo por buscar un nuevo y mejor rumbo empresarial debería pasar por la consideración de estos tres aspectos. Sin embargo, en esta lógica, las personas son las que diseñan las estrategias y las que conforman las organizaciones. Si consideramos que las personas tienen sus propios modelos mentales, que son la base del «gran modelo mental organizacional» que configurará la manera de pensar y hacer las cosas, es decir, la cultura; se puede concluir que la última es la base del funcionamiento y éxito organizacional. Una cultura organizacional positiva permitirá que las estrategias se generen y fluyan de mejor manera.

\section{La gran respuesta a todo es la cultura}

Se cree que la cultura organizacional puede ser uno de los paliativos más importantes para pre- venir o solucionar los grandes problemas en las organizaciones, sean estos puntuales o de mayor envergadura. Sin cultura no hay organización, no hay sentimiento de pertenencia, no hay sentido de comunidad, no hay logros ni celebraciones (Kouzes y Posner 1999), no puede haber aprendizaje ni cambio, ni liderazgo, ni trabajo en equipo ni motivación. Además, solo la cultura permite salir airoso ante problemas que pueda plantear el poder, la política y todo la complejidad que surge al juntar y manejar personas.

En suma, la labor administrativa, desde hace cierto tiempo, ha empezado a preocuparse cada vez más por gestionar desde ópticas diferentes y el punto de vista relacionado con las personas resulta muy relevante. Así, los resultados financieros y lo meramente tangible no son las únicas preocupaciones actuales de los gerentes en el arduo camino de la construcción de estrategias competitivas. 


\section{Referencias}

ARGANDOÑA, Antonio

1998 La teoría de los stakeholders y el bien común. Documento de Investigación Nº 355. IESE, Universidad de Navarra.

BARKER, Joel Arthur

1998 Paradigmas: el negocio de descubrir el futuro. Santafé de Bogotá: McGraw-Hill.

BARNEY, Jay

2002 Gaining and Sustaining Competitive Advantage. Upper Saddle River: Prentice Hall.

BLAKE, Robert R.

1989 Cómo trabajar en equipo: una teoría para impulsar la productividad de su grupo empresarial. Bogotá: Ed. Norma.

BREITFELDER, Matthew D.

2008 «¿Por qué ingresamos a Recursos Humanos?». En: Harvard Business Review América Latina, julio, pp 40-44.

BUENO, Eduardo

1992 Competencia y calidad en Mercado único: la Europa sin fronteras interiores. Madrid: Comunidad Económica Europea.

CANTERA, Francisco Javier y Francisco GIL

2006 Estrategia integral e integrada de gestión de personas. Madrid: Pearson.

CHIAVENATO, Idalberto

1990 Administración de recursos humanos. Bogotá: McGraw-Hill.

2002 Gestión del talento humano. Bogotá: McGrawHill.

DAVID, Fred R.

2007 Conceptos de administración estratégica. 9a. edición. México, D.F.: Pearson Educación.

FREEMAN, R.E.

1984 Strategic Management: A Stakeholder Approach. Boston: Pitman Press.
GOLEMAN, Daniel y Cary CHEMISS

2001 The Emotionally Intelligent Workplace: How to Select for, Measure, and Improve Emotional Intelligence in Individuals, Groups, and Organizations. 1a edición. San Francisco: Jossey-Bass.

GRATTON, Linda

2008 Puntos calientes: qué hace que algunos equipos vibren con energía y otros no. Bogotá: Grupo Editorial Norma.

2001 Estrategias de capital humano. Madrid: Pearson Educación.

HAMEL, Gary y C.K. PARLAD

1996 Competing for the Future: A Breakthrough Strategy for Seizing Control of Your Industry and Dominating the Markets of Tomorrow. Boston: Harvard Business Press.

HELLRIEGEL, Don y Susan JACKSON

2006 Administración: un enfoque basado en competencias. México: Cengage Learning Editores.

HERNÁNDEZ, Gregorio; Claudia ÁLVAREZ y Julia NARANJO

2006 «Gestión humana en las organizaciones, un fenómeno complejo: evolución, retos, tendencias y perspectivas de investigación». En: Cuadernos de Administración, Vol. 19, № 32. Bogotá: Pontificia Universidad Javeriana, pp. 225-254.

JEANNET, Jean-Pierre

2000 Dirección de empresas con mentalidad global. Madrid: Prentice Hall.

KOUZES, J. y B. POSNER

1999 El desafío del liderazgo. Buenos Aires: Editorial Granica.

MINTZBERG, Henry

1991 Mintzberg y la dirección. Madrid: Eds. Díaz de Santos. 
1984 La estructuración de las organizaciones. Barcelona: Ed. Ariel.

NOHRIA, Nitin; Boris GROYSBERG y Linda-Eling LEE

2008 «Motivación de los empleados: un poderoso nuevo modelo». En: Harvard Business Review América Latina, julio, pp 84-91.

\section{ORTEGA, Antonio}

2006 La antigua Roma: valores para el éxito empresarial. España: Pearson Educación S.A.

PEARCE, John y Richard ROBINSON

2004 Strategic Management: Formulation, Implementation, and Control. Boston: McGraw-Hill.

PETERS, Tom y Nancy AUSTIN

1987 Pasión por la excelencia. Barcelona: Folio.
PORTER, Michael

2006 Ventaja competitiva. Creación y sostenimiento de un desempeño superior. $5^{\mathrm{a}}$ reimpresión. México: CECSA.

1996 «What is Strategy». En: Harvard Business Review, noviembre - diciembre, pp. 61-78.

1980 Competitive Strategy. Nueva York: Free Press.

RIES, Al

1992 Posicionamiento: el concepto que ha revolucionado la comunicación publicitaria y la mercadotecnia. Bogotá: McGraw-Hill.

ROBBINS, Stephen P.

2004 Comportamiento organizacional: teoría y práctica. 7a. edición. México: Prentice-Hall Hispanoamericana.

ROBERTS, Kevin

2005 Lovemarks: el futuro más allá de las marcas. Barcelona: Ediciones Urano. 
Anexo 1

Un modelo de gestión que incorpora a las personas como elemento fundamental de la estrategia empresarial

\begin{tabular}{|c|c|c|c|c|}
\hline $\begin{array}{l}\quad \text { IDEAS EJE } \\
\text { - La importancia de la } \\
\text { Visión, la Misión y } \\
\text { los Valores } \\
\text { - La importancia de la } \\
\text { selección de las } \\
\text { personas } \\
\text { - El Paso de la concepción } \\
\text { de "Recursos Humanos" } \\
\text { a "Personas" y de allí, } \\
\text { a "Talentos". } \\
\text { - Las Personas son } \\
\text { fuente de Ventaja } \\
\text { Competitiva } \\
\text { - La competencia del } \\
\text { futuro es cada vez màs } \\
\text { por intangibles, } \\
\text { las personas son } \\
\text { "extrañas" porque } \\
\text { elevadas a la "n” } \\
\text { pueden ser un intangible } \\
\text { diferenciador y a la vez, } \\
\text { son las que gestionan } \\
\text { esta nueva realidad. } \\
\text { - Las personas deben } \\
\text { ser lo más importante } \\
\text { para las empresas. }\end{array}$ & \begin{tabular}{|l|} 
\\
PUNTOS CLAVE \\
- El modelo necesita \\
una \\
retroalimentación \\
constante y está \\
evolucionando. \\
- La dicotomía de \\
lo burocrático \\
ante lo orgánico \\
- El compromiso \\
del ápice \\
estratégico
\end{tabular} & $\begin{array}{l}\text { ASPECTOS A } \\
\text { GERENCIAR } \\
\text { - Se está } \\
\text { gerenciando } \\
\text { personas. } \\
\text { - CULTURA, } \\
\text { Liderazgo, } \\
\text { Motivación y } \\
\text { Trabajo en } \\
\text { Equipo } \\
\\
\text { Aspectos que } \\
\text { alteran la } \\
\text { eficiencia de la } \\
\text { gestión de } \\
\text { personas: el } \\
\text { Poder y la } \\
\text { Política } \\
\text { - Aspectos que } \\
\text { se busca } \\
\text { lograr: } \\
\text { Aprendizaje y } \\
\text { Cambio. }\end{array}$ & $\begin{array}{l}\text { ¿CÓMO SE } \\
\text { PUEDE } \\
\text { GERENCIAR } \\
\text { EFICIENTE- } \\
\text { MENTE A LAS } \\
\text { ORGANIZA- } \\
\text { CIONES DEL } \\
\text { NUEVO } \\
\text { MILENIO? } \\
\\
\text { - Pensando antes } \\
\text { de actuar } \\
\text { - Rompiendo } \\
\text { Paradigmas } \\
\text { - Generando } \\
\text { Cultura } \\
\text { - Aprendiendo y } \\
\text { Cambiando } \\
\text { - Gestionando el } \\
\text { Tiempo } \\
\text { - Generando } \\
\text { diálogo }\end{array}$ & $\begin{array}{c}\text { EFICIENCIA } \\
\text { ORGANIZACIONAL } \\
\text { SOBRE LA BASE DE LA } \\
\text { CONSTRUCCIÓN DE } \\
\text { UNA CULTURA } \\
\text { ORGANIZACIONAL } \\
\text { POSITIVA Y FUERTE }\end{array}$ \\
\hline
\end{tabular}

Elaboración Propia 


\section{Anexo 2}

Las 25 mejores empresas para trabajar en el perú según el great pleace to work perú - año 2007

\begin{tabular}{|c|c|c|}
\hline Puesto & Empresa & Industria \\
\hline 1 & Kimberly Clark Perú & Manufactura y Producción - Cuidado de la salud. \\
\hline 2 & IBM del Perú & Información Tecnológica (IT) \\
\hline 3 & Microsoft Perú & Información Tecnológica (IT) \\
\hline 4 & Pfizer & Biotecnología y Farmacia - Farmacia \\
\hline 5 & J \& V Resguardo & Servicios profesionales - Seguridad \\
\hline 6 & 3M Perú & Retail - Venta al por menor \\
\hline 7 & Interbank & Servicios Financieros y Seguros - Bancos/Servicios de crédito \\
\hline 8 & Hewlett Packard Perú & Información Tecnológica (IT) \\
\hline 9 & Productos Roche & Biotecnología y Farmacia - Farmacia \\
\hline 10 & CinePlanet & Hospitalidad \\
\hline 11 & Interseguro & Servicios Financieros y Seguros - Seguros generales \\
\hline 12 & Hipermercado Tottus & Retail - Venta al por menor \\
\hline 13 & McDonald's & Hospitalidad - Servicios de comida y bebida \\
\hline 14 & Telefónica Centros de Cobro & Servicios profesionales - Call center \\
\hline 15 & Cetco & Retail - Venta al por menor \\
\hline 16 & Banco Interamericano de Finanzas & Servicios Financieros y Seguros - Bancos/Servicios de crédito \\
\hline 17 & Sodimac Perú & Retail - Venta al por menor - Especialidades \\
\hline 18 & Corporación Cormin & Servicios Industriales \\
\hline 19 & British American Tobacco del Perú & Retail - Venta al por menor \\
\hline 20 & Pacífico Seguros & Servicios Financieros y Seguros - Seguros generales \\
\hline 21 & Duke Energy Egenor & Manufactura y Producción - Distribución de energía \\
\hline 22 & Banco de Crédito del Perú & Servicios Financieros y Seguros - Bancos/Servicios de crédito \\
\hline 23 & $\begin{array}{l}\text { Compañía Peruana de Medios de Pago } \\
\text { (VisaNet) }\end{array}$ & Servicios Financieros y Seguros - Bancos/Servicios de crédito \\
\hline 24 & Natura Cosméticos & Retail - Venta al por menor \\
\hline 25 & Lima Tours & Servicios profesionales - Agencia de viajes \\
\hline
\end{tabular}

Fuente: http://www.greatplacetowork.com.pe 